\title{
Some Characterization Results on Dynamic Cumulative Residual Tsallis Entropy
}

\author{
Madan Mohan Sati ${ }^{1}$ and Nitin Gupta ${ }^{2}$ \\ ${ }^{1}$ Department of Mathematics, Jaypee University of Information Technology, Waknaghat, Solan, Himachal Pradesh 173234, India \\ ${ }^{2}$ Department of Mathematics, Indian Institute of Technology Kharagpur, Kharagpur 721302, India
}

Correspondence should be addressed to Madan Mohan Sati; madan25mohansati@gmail.com

Received 5 June 2015; Revised 7 September 2015; Accepted 7 September 2015

Academic Editor: Zacharias Psaradakis

Copyright (c) 2015 M. M. Sati and N. Gupta. This is an open access article distributed under the Creative Commons Attribution License, which permits unrestricted use, distribution, and reproduction in any medium, provided the original work is properly cited.

We propose a generalized cumulative residual information measure based on Tsallis entropy and its dynamic version. We study the characterizations of the proposed information measure and define new classes of life distributions based on this measure. Some applications are provided in relation to weighted and equilibrium probability models. Finally the empirical cumulative Tsallis entropy is proposed to estimate the new information measure.

\section{Introduction}

Shannon [1] introduced the concept of entropy which is widely used in the fields of communication theory, information theory, physics, economics, probability and statistics, and so forth.

Let $X$ be a random variable having probability density function $f(x)$, survival function $\bar{F}(x)$, and hazard rate $r(x)=$ $f(x) / \bar{F}(x)$; Shannon defined entropy for a random variable $X$ as

$$
H(X)=-\int_{0}^{\infty} f(x) \log (f(x)) d x .
$$

For a residual lifetime $X_{t}=[X-t \mid X>t]$, where $t>$ 0 , Ebrahimi [2] defined an entropy as a dynamic measure of uncertainty which is given by

$$
H(X ; t)=-\int_{t}^{\infty} \frac{f(x)}{\bar{F}(t)} \log \left(\frac{f(x)}{\bar{F}(t)}\right) d x .
$$

Alternative entropy was introduced by Rao et al. [3] which is based on survival function instead of probability density function. Rao et al. [3] defined entropy as

$$
\xi(X)=-\int_{0}^{\infty} \bar{F}(x) \log (\bar{F}(x)) d x
$$

and called it the cumulative residual entropy (CRE).
For the study of the properties and applications of CRE, we refer to Asadi and Ebrahimi [4], Asadi et al. [5], Rao [6], Drissi et al. [7], Gupta [8], Di Crescenzo and Longobardi [9], Navarro et al. [10], Gupta and Taneja [11], and Taneja and Kumar [12].

For a residual lifetime $X_{t}=[X-t \mid X>t]$, Asadi and Zohrevand [13] defined the dynamic measure of CRE as

$$
\xi(X ; t)=-\int_{t}^{\infty} \frac{\bar{F}(x)}{\bar{F}(t)} \log \left(\frac{\bar{F}(x)}{\bar{F}(t)}\right) d x
$$

and called it as dynamic cumulative residual entropy (DCRE).

Abbasnejad et al. [14] proposed dynamic survival entropy of order $\alpha$ and gave the relation of dynamic survival entropy with the mean residual life function. Further Sunoj and Linu [15] defined cumulative residual Renyi entropy of order $\beta$ and its dynamic version. Recently Kumar and Taneja [16] defined generalized cumulative residual information measure and its dynamic version based on Varma's entropy function.

Renyi [17] defined the generalized entropy of order $\alpha$ as

$$
H_{\alpha}(X)=\frac{1}{1-\alpha} \log \left(\int_{0}^{\infty}(f(x))^{\alpha} d x\right)
$$


Tsallis [18] defined the generalized entropy of order $\alpha$ as

$$
S_{\alpha}(X)=\frac{1}{\alpha-1}\left(1-\int_{0}^{\infty}(f(x))^{\alpha} d x\right),
$$

$\alpha>0, \alpha \neq 1$.

Both entropies (5) and (6) approach the Shannon entropy as $\alpha \rightarrow 1$. There is a close relationship between the Renyi entropy and the Tsallis entropy given as

$$
H_{\alpha}(X)=\frac{1}{(\alpha-1)} \log \left[1-(\alpha-1) S_{\alpha}(X)\right] .
$$

It may be noted that Tsallis entropy is a nonextensive entropy and it is nonlogarithmic. However, Renyi entropy is an extensive entropy which is the major difference between them (cf. $[19,20])$.

Tsallis entropy plays a central role in different areas such as physics, chemistry, biology, medicine, and economics. Cartwright [21] proposed applications of Tsallis entropy in various fields such as describing the fluctuation of magnetic field in solar wind, signs of breast cancer in mammograms, atoms in optical lattices, analysis in magnetic resonance imaging (MRI).

The aim of the paper is to study the cumulative residual information based on nonextensive entropy measures and characterize some well known lifetime distributions and probability models. The empirical form of this information measure is useful for real data problems. In Section 2, we propose a cumulative residual entropy based on Tsallis entropy of order $\alpha$ and its dynamic version. Also, we study some characterization results using the relationship of dynamic cumulative residual Tsallis entropy (DCRTE) with hazard rate function and mean residual life function. In Section 3, we define new classes of life distribution based on these measures. In Section 4, we propose the weighted form of DCRTE and study its various properties. In Section 5 we introduce the empirical cumulative Tsallis entropy and express it in terms of the sample spacings. In order to study the empirical cumulative Tsallis entropy, an example is also being provided.

\section{Dynamic Cumulative Residual Tsallis Entropy (DCRTE)}

In this section, we define the cumulative residual Tsallis entropy and dynamic cumulative residual Tsallis entropy. We also give some characterization results of well known distributions in terms of DCRTE.

Definition 1. For a random variable $X$ with survival function (sf) $\bar{F}(x)$, the cumulative residual entropy of order $\alpha$ denoted by $\eta_{\alpha}(X)$ is defined as

$$
\eta_{\alpha}(X)=\frac{1}{\alpha-1}\left(1-\int_{0}^{\infty}(\bar{F}(x))^{\alpha} d x\right),
$$

$\alpha>0, \alpha \neq 1$

Letting $\alpha \rightarrow 1$, (8) gives

$$
\lim _{\alpha \rightarrow 1} \eta_{\alpha}(X)=\xi(X) \text {. }
$$

Let us consider a unit whose random life is represented by random variable $X$. Let the unit survive up to time $t$; then the information based on entropy of the random variable $X$ may not be useful. In that case we may consider dynamic (time dependent) information based on the entropy of the random variable $X_{t}=[X-t \mid X>t]$. The random variable $X_{t}=$ $[X-t \mid X>t]$ has the survival function

$$
\bar{F}_{t}(x)= \begin{cases}\bar{F}(x) & \text { where } x>t \\ \bar{F}(t) & \text { otherwise. }\end{cases}
$$

Definition 2. For a random variable $X_{t}$ with survival function $\bar{F}(x)$, the dynamic cumulative residual entropy of order $\alpha$ denoted by $\eta_{\alpha}(X ; t)$ is defined as

$$
\begin{aligned}
& \eta_{\alpha}(X ; t)=\frac{1}{\alpha-1}\left(1-\int_{t}^{\infty}\left(\frac{\bar{F}(x)}{\bar{F}(t)}\right)^{\alpha} d x\right) \\
& \alpha>0, \alpha \neq 1 .
\end{aligned}
$$

The following theorem shows that the dynamic cumulative residual entropy determines the survival function $\bar{F}(x)$ uniquely.

Theorem 3. Let the nonnegative random variable $X$ have the density function $f(x)$, the survival function $\bar{F}(x)$, and the hazard rate $r(x)$. Assume that $\eta_{\alpha}(X ; t)<\infty ; t \geq 0 ; \forall \alpha>$ $0(\neq 1)$. Then for each $\alpha, \eta_{\alpha}(X ; t)$ (whereas $\eta_{\alpha}^{\prime}(X ; t) \neq 0$ ) uniquely determines the survival function $\bar{F}(t)$.

Proof. Consider the dynamic cumulative residual Tsallis entropy of order $\alpha$ as (11). Therefore, we have

$$
(\alpha-1) \eta_{\alpha}(X ; t)=1-\frac{\int_{t}^{\infty}(\bar{F}(x))^{\alpha} d x}{(\bar{F}(t))^{\alpha}} .
$$

Differentiating (12) with respect to $t$, we have

$$
\begin{aligned}
(\alpha-1) \eta_{\alpha}^{\prime}(X ; t) & =1-\alpha f(t) \frac{\int_{t}^{\infty}(\bar{F}(x))^{\alpha} d x}{(\bar{F}(t))^{\alpha+1}} \\
& =1+\alpha r(t)\left((\alpha-1) \eta_{\alpha}(X ; t)-1\right),
\end{aligned}
$$

where the last step follows from (12) and $r(x)=f(x) / \bar{F}(x)$ is the hazard rate function of random variable $X$. Consider two survival functions $\bar{F}_{1}(t)$ and $\bar{F}_{2}(t)$ having dynamic entropies as $\eta_{\alpha}\left(X_{1} ; t\right)$ and $\eta_{\alpha}\left(X_{2} ; t\right)$ and hazard rate functions $r_{1}(t)$ and $r_{2}(t)$, respectively. Letting $\eta_{\alpha}\left(X_{1} ; t\right)=\eta_{\alpha}\left(X_{2} ; t\right)$ implies that $\eta_{\alpha}^{\prime}\left(X_{1} ; t\right)=\eta_{\alpha}^{\prime}\left(X_{2} ; t\right)$ which further implies

$$
\begin{aligned}
& (\alpha-1) \eta_{\alpha}^{\prime}\left(X_{1} ; t\right)=(\alpha-1) \eta_{\alpha}^{\prime}\left(X_{2} ; t\right), \\
& \text { i.e., } 1+\alpha r_{1}(t)\left((\alpha-1) \eta_{\alpha}\left(X_{1} ; t\right)-1\right) \\
& \quad=1+\alpha r_{2}(t)\left((\alpha-1) \eta_{\alpha}\left(X_{2} ; t\right)-1\right) ;
\end{aligned}
$$

last step follows using (13). Now as $\eta_{\alpha}\left(X_{1} ; t\right)=\eta_{\alpha}\left(X_{2} ; t\right)$, therefore, (14) reduces to $r_{1}(t)=r_{2}(t)$ or equivalently $\bar{F}_{1}(t)=$ $\bar{F}_{2}(t)$. 
Now we provide some characterization results in terms of relationship between DCRTE and hazard rate function $r(t)$.

Theorem 4. Let $X$ be a nonnegative continuous random variable with survival function $\bar{F}(t)$ hazard rate function $r(t)$ and dynamic cumulative residual Tsallis entropy $\eta_{\alpha}(X ; t)$; then the relationship,

$$
(\alpha-1) \eta_{\alpha}^{\prime}(X ; t)=c r(t), \quad \alpha>0, \alpha \neq 1,
$$

gives survival function $\bar{F}(t)=\exp \left\{-\int_{0}^{t}(1 / \sqrt{K-2 \alpha c x}) d x\right\}$, where $K$ is the constant of integration and characterizes

(i) an exponential distribution for $K>0$ and $c=0$ with survival function

$$
\bar{F}(t)=e^{-\lambda t}, \quad \lambda>0, t>0
$$

and

(ii) Weibull distribution for $K=0$ and $c<0$ with survival function

$$
\bar{F}(t)=e^{-(\gamma t)^{\beta}}, \quad \gamma>0, \beta=\frac{1}{2}, t>0 .
$$

Proof. Under the assumption that (15) holds; using (13), we have

$$
1+\alpha r(t)\left\{(\alpha-1) \eta_{\alpha}(X ; t)-1\right\}=c r(t)
$$

or equivalently, using (12),

$$
\frac{\bar{F}(t)}{f(t)}-c=\alpha\left\{\frac{\int_{t}^{\infty} \bar{F}^{\alpha}(x) d x}{\bar{F}^{\alpha}(t)}\right\} .
$$

Differentiating (19) with respect to $t$, we have

$$
\begin{aligned}
& -\left[1+\frac{\bar{F}(t)}{(f(t))^{2}} f^{\prime}(t)\right] \\
& =\alpha\left[-1+\alpha \frac{f(t)}{\bar{F}(t)}\left(\frac{\int_{t}^{\infty} \bar{F}^{\alpha}(x) d x}{\bar{F}^{\alpha}(t)}\right)\right], \\
& -\left[1+\frac{1}{r(t)} \cdot \frac{f^{\prime}(t)}{f(t)}\right]=\alpha\left[-1+r(t)\left(\frac{\bar{F}(t)}{f(t)}-c\right)\right], \\
& {\left[1+\frac{1}{r(t)} \cdot \frac{f^{\prime}(t)}{f(t)}\right]=\alpha c r(t),} \\
& \alpha c(r(t))^{2}-r(t)=\frac{f^{\prime}(t)}{f(t)}, \\
& \alpha c(r(t))^{2}=\frac{d}{d t}(\log r(t)) .
\end{aligned}
$$

Now letting $\log (r(t))=y$, that is, $r(t)=e^{y},(20)$ reduces to

$$
\begin{aligned}
\alpha c e^{2 y} & =\frac{d y}{d t}, \\
\text { i.e., } e^{-2 y} d y & =\alpha c d t .
\end{aligned}
$$

Integrating on both sides, we have

$$
e^{-2 y}=K-2 \alpha c t,
$$

where $K$ is a constant. Therefore, $r(t)=e^{y}$ provides

$$
r(t)=\frac{1}{\sqrt{K-2 \alpha c t}} .
$$

Since the hazard rate uniquely determines the survival function using the relationship $\bar{F}(t)=\exp \left\{-\int_{0}^{t} r(x) d x\right\}$, consider the following case.

(i) For $K>0$ and $c=0, \bar{F}(t)=\exp \left\{-\int_{0}^{t}(1 / \sqrt{K}) d x\right\}$ or equivalently $\bar{F}(t)=e^{-\lambda t}$, where $\lambda=1 / \sqrt{K}>0$.

(ii) For $c \neq 0$, survival function $\bar{F}(t)=e^{-\sqrt{K} / \alpha c} e^{\sqrt{K-2 \alpha c t} / \alpha c}$. Further, for $K=0$ and $c<0(c=-a, a>0), \bar{F}(t)=$ $e^{-(\gamma t)^{\beta}}$, where $\gamma=2 / a \alpha>0, \beta=1 / 2>0$.

Converse. We assume that random variable $X$ is distributed exponentially with pdf $\lambda e^{-\lambda x}, \lambda>0, x \geq 0$. Using (12), we have $(\alpha-1) \eta_{\alpha}(X ; t)=(1-1 / \alpha \lambda)$ from which (15) follows with $c=0$.

When $X$ is distributed as Weibull with $\operatorname{pdf} \beta \gamma^{\beta} x^{\beta-1} e^{-(\gamma x)^{\beta}}$, $\gamma>0, \beta=1 / 2, x \geq 0$. Using (12), we have $(\alpha-1) \eta_{\alpha}(X ; t)=$ $1-\left(2 / \alpha^{2} \gamma\right)\left(1+\alpha(\gamma t)^{1 / 2}\right)$ which on differentiation yields $(\alpha-$ 1) $\eta_{\alpha}^{\prime}(X ; t)=-1 / \alpha(\gamma t)^{1 / 2}=\operatorname{cr}(t)$ with $r(t)=(1 / 2)(\gamma / t)^{1 / 2}$ and $c=-a<0$.

The following theorem characterize the distributions using relationship between DCRTE and mean residual life (MRL) $m_{F}(t)$.

Theorem 5. Let $X$ be a nonnegative continuous random variable with survival function $\bar{F}(t), M R L m_{F}(t)$, and dynamic cumulative residual Tsallis entropy of order $\alpha, \eta_{\alpha}(X ; t)$; if

$$
(\alpha-1) \eta_{\alpha}(X ; t)=1-K m_{F}(t), \quad \alpha>0, \alpha \neq 1,
$$

then X has

(i) an exponential distribution iff $K=1 / \alpha$,

(ii) a Pareto distribution iff $K<1 / \alpha$,

(iii) a finite range distribution iff $K>1 / \alpha$.

Proof. (i) If random variable $X$ denotes an exponential distribution, then it has pdf, sf, and MRL, respectively, as

$$
\begin{aligned}
f(t) & =\lambda e^{-\lambda t}, \quad \lambda>0, t>0, \\
\bar{F}(t) & =e^{-\lambda t}, \\
m_{F}(t) & =\frac{1}{\lambda} .
\end{aligned}
$$

Therefore, after simplification, using (12),

$$
(\alpha-1) \eta_{\alpha}(X ; t)=1-K m_{F}(t),
$$

where $K=1 / \alpha$ and $m_{F}(t)=1 / \lambda$. 
(ii) If random variable $X$ denotes the Pareto distribution, then it has pdf, sf, and MRL, respectively, as

$$
\begin{aligned}
f(t) & =\left(1+\frac{t}{a}\right)^{-a-1}, \quad a>1, t>0, \\
\bar{F}(t) & =\left(1+\frac{t}{a}\right)^{-a}, \\
m_{F}(t) & =\frac{(a+t)}{(a-1)} .
\end{aligned}
$$

Therefore, after simplification, using (12),

$$
(\alpha-1) \eta_{\alpha}(X ; t)=1-K m_{F}(t)
$$

where $K=(a-1) /(\alpha a-1)<1 / \alpha$ if $\alpha>1$ and $m_{F}(t)=$ $(a+t) /(a-1)$.

(iii) If random variable $X$ denotes the finite range distribution, then it has pdf, sf, and MRL, respectively, as

$$
\begin{aligned}
f(t) & =b(1-t)^{b-1}, \quad b>0,0<t<1, \\
\bar{F}(t) & =(1-t)^{b}, \\
m_{F}(t) & =\frac{(1-t)}{(b+1)} .
\end{aligned}
$$

Therefore, after simplification, using (12),

$$
(\alpha-1) \eta_{\alpha}(X ; t)=1-K m_{F}(t),
$$

where $K=(b+1) /(\alpha b+1)>1 / \alpha$ if $\alpha>1$ and $m_{F}(t)=$ $(1-t) /(b+1)$.

Converse. Let (24) holds. Using (12), we get

$$
K m_{F}(t)=\frac{\int_{t}^{\infty}(\bar{F}(x))^{\alpha} d x}{(\bar{F}(t))^{\alpha}} ;
$$

differentiating (31) with respect to $t$, we obtain

$$
\begin{aligned}
K m_{F}^{\prime}(t) & =-1+\alpha r(t) \frac{\int_{t}^{\infty}(\bar{F}(x))^{\alpha} d x}{(\bar{F}(t))^{\alpha}} \\
& =-1+\alpha r(t) K m_{F}(t) .
\end{aligned}
$$

Using the relation between mean residual life and hazard rate, that is, $r(t) m_{F}(t)=1+m_{F}^{\prime}(t)$, we have

$$
m_{F}^{\prime}(t)=\frac{(K \alpha-1)}{K(1-\alpha)} .
$$

Integrating (32) on both sides with respect to $t$ over $(0, x)$, we get

$$
m_{F}(x)=\frac{(K \alpha-1)}{K(1-\alpha)} x+m_{F}(0) .
$$

Equation (33) is linear in MRL function $m_{F}(x)$ of continuous random variable $X$, if and only if the underlying distribution is exponential $(K=1 / \alpha)$, Pareto $(K<1 / \alpha)$, or finite range $(K>1 / \alpha)$; refer to Hall and Wellner [22]. This completes the theorem.

\section{New Class of Life Distributions}

In this section, we define new class of life distribution based on the DCRTE $\eta_{\alpha}(X ; t)$.

Definition 6. The distribution function $F$ is said to be increasing (decreasing) DCRTE, denoted by IDCRTE (DDCRTE), if $\eta_{\alpha}(X ; t)$ is an increasing (decreasing) function of $t$.

The following theorem gives the necessary and sufficient condition for $\eta_{\alpha}(X ; t)$ to be increasing (decreasing) DCRTE.

Theorem 7. The distribution function $F$ is increasing (decreasing) DCRTE if and only if for all $t \geq 0$

$$
\eta_{\alpha}(X ; t) \geq(\leq) \frac{1}{(\alpha-1)}\left(1-\frac{1}{\alpha r(t)}\right), \quad \forall \alpha>0(\neq 1) .
$$

Proof. The proof of the theorem directly follows from (13).

In the following theorem, we give the hazard rate ordering using the DCRTE.

Theorem 8. Let $X$ and $Y$ be two nonnegative absolutely continuous random variables with survival functions $\bar{F}(t)$ and $\bar{G}(t)$ and hazard rate functions $r_{F}(t)$ and $r_{G}(t)$, respectively. If $X \geq{ }^{h r} Y$, that is, $r_{F}(t) \leq r_{G}(t)$ for all $t \geq 0$, then

$$
\eta_{\alpha}(X ; t) \leq(\geq) \eta_{\alpha}(Y ; t), \quad \forall \alpha>1 \quad(0<\alpha<1) .
$$

Proof. The assumption that $r_{F}(t) \leq r_{G}(t)$ implies $\bar{F}_{X_{t}}(x) \geq$ $\bar{G}_{X_{t}}(x)$ :

$$
\begin{array}{r}
\left(\frac{\bar{F}(x)}{\bar{F}(t)}\right)^{\alpha} \geq\left(\frac{\bar{G}(x)}{\bar{G}(t)}\right)^{\alpha}, \quad \forall \alpha>0, \\
1-\int_{t}^{\infty}\left(\frac{\bar{F}(x)}{\bar{F}(t)}\right)^{\alpha} d x \leq 1-\int_{t}^{\infty}\left(\frac{\bar{G}(x)}{\bar{G}(t)}\right)^{\alpha} d x .
\end{array}
$$

For $\alpha>1$

$$
\begin{aligned}
& \frac{1}{(\alpha-1)}\left(1-\int_{t}^{\infty}\left(\frac{\bar{F}(x)}{\bar{F}(t)}\right)^{\alpha} d x\right) \\
& \leq \frac{1}{(\alpha-1)}\left(1-\int_{t}^{\infty}\left(\frac{\bar{G}(x)}{\bar{G}(t)}\right)^{\alpha} d x\right), \\
& \eta_{\alpha}(X ; t) \leq \eta_{\alpha}(Y ; t) .
\end{aligned}
$$

For $0<\alpha<1$

$$
\begin{aligned}
& \frac{1}{(1-\alpha)}\left(\int_{t}^{\infty}\left(\frac{\bar{F}(x)}{\bar{F}(t)}\right)^{\alpha} d x-1\right) \\
& \geq \frac{1}{(1-\alpha)}\left(\int_{t_{1}}^{\infty}\left(\frac{\bar{G}(x)}{\bar{G}(t)}\right)^{\alpha} d x-1\right), \\
& \eta_{\alpha}(X ; t) \geq \eta_{\alpha}(Y ; t) .
\end{aligned}
$$


Hence

$$
\eta_{\alpha}(X ; t) \leq(\geq) \eta_{\alpha}(Y ; t), \quad \forall \alpha>1(0<\alpha<1) .
$$

In the following lemma, we discuss the effect of linear transformation on DCRTE.

Lemma 9. For any nonnegative random variable $X$, let $Z=$ $a X+b$, where $a>0$ and $b \geq 0$; then

$$
\eta_{\alpha}(Z ; t)=\frac{(1-a)}{(\alpha-1)}+a \eta_{\alpha}\left(X ; \frac{t-b}{a}\right), \quad t \geq b .
$$

Proof. The result follows using the fact that $\bar{F}_{a X+b}(x)=$ $\bar{F}_{X}((x-b) / a), x \in R$ and (11).

\section{Weighted Dynamic Cumulative Residual Tsallis Entropy}

Let $X$ be a random variable with probability density function $f(t)$ and survival function $\bar{F}(t)$. Let $X_{W}$ be weighted random variable associated with $X$ and their probability density function and survival function denoted by $f_{w}(t)$ and $\bar{F}_{w}(t)$, given by

$$
\begin{aligned}
f_{w}(t) & =\frac{w(t) f_{X}(t)}{E(w(X))}, \\
\bar{F}_{w}(t) & =\frac{E(w(X) \mid X>t) \bar{F}_{X}(t)}{E(w(X))} ; \\
& 0<E(w(X))<\infty .
\end{aligned}
$$

The weighted dynamic cumulative residual Tsallis entropy denoted by $\eta_{\alpha}(W ; t)$ is proposed as

$$
\begin{aligned}
\eta_{\alpha}(W ; t)=\frac{1}{(\alpha-1)}\left(1-\int_{t}^{\infty}\left(\frac{\bar{F}_{w}(x)}{\bar{F}_{w}(t)}\right)^{\alpha} d t\right), \\
\alpha>0(\neq 1) .
\end{aligned}
$$

The importance of weighted distribution can be seen in Patil and Rao [23], Gupta and Kirmani [24], Nair and Sunoj [25], Di Crescenzo and Longobardi [26], and Maya and Sunoj [27]. For the weighted distribution, we obtain the following result based on MRL ordering.

Theorem 10. If $E(w(X) \mid X>x) \leq E(w(X) \mid X>t)$ for all $x \geq t$, then $\eta_{\alpha}(W ; t) \leq(\geq) \eta_{\alpha}(X ; t)$ for $0<\alpha<1(\alpha>1)$. If $E(w(X) \mid X>x) \geq E(w(X) \mid X>t)$ for all $x \geq t$, then $\eta_{\alpha}(W ; t) \geq(\leq) \eta_{\alpha}(X ; t)$ for $0<\alpha<1(\alpha>1)$.

Proof. Rewriting (43), we have

$$
\begin{aligned}
& \eta_{\alpha}(W ; t)=\frac{1}{(\alpha-1)}\left(1-\int_{t}^{\infty}\left(\frac{\bar{F}_{w}(x)}{\bar{F}_{w}(t)}\right)^{\alpha} d x\right) \\
& =\frac{1}{(\alpha-1)}(1 \\
& \left.\quad-\int_{t}^{\infty} \frac{[E(w(X) \mid X>x) \bar{F}(x)]^{\alpha}}{[E(w(X) \mid X>t) \bar{F}(t)]^{\alpha}} d x\right)
\end{aligned}
$$

$$
\begin{aligned}
= & \frac{1}{(\alpha-1)}(1 \\
& \left.-\int_{t}^{\infty}\left[\frac{E(w(X) \mid X>x)}{E(w(X) \mid X>t)}\right]^{\alpha}\left(\frac{\bar{F}^{\alpha}(x)}{\bar{F}^{\alpha}(t)}\right) d x\right) .
\end{aligned}
$$

If $E(w(X) \mid X>x) \leq E(w(X) \mid X>t)$ for all $x \geq t$, that is, $[E(w(X) \mid X>x) / E(w(X) \mid X>t)] \leq 1$ for all $x \geq t$, then we have

$$
\begin{aligned}
& \int_{t}^{\infty}\left[\frac{E(w(X) \mid X>x)}{E(w(X) \mid X>t)}\right]^{\alpha}\left(\frac{\bar{F}^{\alpha}(x)}{\bar{F}^{\alpha}(t)}\right) d x \\
& \quad \leq \int_{t}^{\infty}\left(\frac{\bar{F}^{\alpha}(x)}{\bar{F}^{\alpha}(t)}\right) d x,
\end{aligned}
$$

which further implies the following: for all $0<\alpha<1(\alpha>1)$,

$$
\begin{aligned}
& \frac{1}{(1-\alpha)} \int_{t}^{\infty}\left[\frac{E(w(X) \mid X>x)}{E(w(X) \mid X>t)}\right]^{\alpha}\left(\frac{\bar{F}^{\alpha}(x)}{\bar{F}^{\alpha}(t)}\right) d x \\
& \leq(\geq) \frac{1}{(1-\alpha)} \int_{t}^{\infty}\left(\frac{\bar{F}^{\alpha}(x)}{\bar{F}^{\alpha}(t)}\right) d x, \\
& \frac{1}{(\alpha-1)}+\frac{1}{(1-\alpha)} \\
& \cdot \int_{t}^{\infty}\left[\frac{E(w(X) \mid X>x)}{E(w(X) \mid X>t)}\right]^{\alpha}\left(\frac{\bar{F}^{\alpha}(x)}{\bar{F}^{\alpha}(t)}\right) d x \\
& \leq(\geq) \frac{1}{(\alpha-1)}+\frac{1}{(1-\alpha)} \int_{t}^{\infty}\left(\frac{\bar{F}^{\alpha}(x)}{\bar{F}^{\alpha}(t)}\right) d x, \\
& \frac{1}{(\alpha-1)}-\frac{1}{(\alpha-1)} \\
& \cdot \int_{t}^{\infty}\left[\frac{E(w(X) \mid X>x)}{E(w(X) \mid X>t)}\right]^{\alpha}\left(\frac{\bar{F}^{\alpha}(x)}{\bar{F}^{\alpha}(t)}\right) d x \\
& \left.\leq(\geq) \frac{1}{(\alpha-1)}-\frac{1}{(\alpha-1)}\right]_{t}^{\infty}\left(\frac{\bar{F}^{\alpha}(x)}{\bar{F}^{\alpha}(t)}\right) d x, \\
& \quad 1 \\
& \quad \leq(\geq) \frac{1}{(\alpha-1)}\left(1-\int_{t}^{\infty}\left(\frac{\bar{F}^{\alpha}(x)}{\bar{F}^{\alpha}(t)}\right) d x\right) . \\
& \left.\quad-\int_{t}^{\infty}\left[\frac{E(w(X) \mid X>x)}{E(w(X) \mid X>t)}\right]^{\alpha}\left(\frac{\bar{F}^{\alpha}(x)}{\bar{F}^{\alpha}(t)}\right) d x\right)
\end{aligned}
$$

Now using (44) and (11), from (46) we get

$$
\eta_{\alpha}(W ; t) \leq(\geq) \eta_{\alpha}(X ; t), \quad \forall 0<\alpha<1 \quad(\alpha>1) .
$$

Similarly if $E(w(X) \mid X>x) \geq E(w(X) \mid X>t)$ for all $x \geq t$, then

$$
\eta_{\alpha}(W ; t) \geq(\leq) \eta_{\alpha}(X ; t), \quad \forall 0<\alpha<1 \quad(\alpha>1) .
$$


Particularly, when the weight function is defined as $w(t)=\bar{F}(t) / f(t)$, the corresponding weighted distribution becomes the equilibrium distribution. Let $X_{E}$ be a random variable corresponding to equilibrium distribution with probability density function $f_{E}(t)=\bar{F}(t) / \mu, t>0$, and survival function $\bar{F}_{E}(t)=(r(t) / \mu) \bar{F}(t)$, where $\mu=E(X)<\infty$; then dynamic cumulative residual Tsallis entropy of $X_{E}$ is proposed as

$$
\begin{aligned}
\eta_{\alpha}(E ; t)=\frac{1}{(\alpha-1)}\left(1-\int_{t}^{\infty}\left(\frac{\bar{F}_{E}(x)}{\bar{F}_{E}(t)}\right)^{\alpha} d x\right), & \\
\alpha & >0(\neq 1) .
\end{aligned}
$$

Theorem 11. If $\bar{F}(t)$ has decreasing hazard rate, then $\eta_{\alpha}(E ; t) \geq$ $(\leq) \eta_{\alpha}(X ; t)$ for $0<\alpha<1(\alpha>1)$. If $\bar{F}(t)$ has increasing hazard rate, then $\eta_{\alpha}(E ; t) \leq(\geq) \eta_{\alpha}(X ; t)$ for $0<\alpha<1(\alpha>1)$.

Proof. Note that

$$
\begin{aligned}
\eta_{\alpha}(E ; t) & =\frac{1}{(\alpha-1)}\left(1-\int_{t}^{\infty}\left(\frac{\bar{F}_{E}(x)}{\bar{F}_{E}(t)}\right)^{\alpha} d x\right) \\
& \alpha>0(\neq 1) \\
& =\frac{1}{(\alpha-1)}\left(1-\int_{t}^{\infty}\left[\frac{r(x)}{r(t)}\right]^{\alpha} \frac{\bar{F}^{\alpha}(x)}{\bar{F}^{\alpha}(t)} d x\right) .
\end{aligned}
$$

Now if $\bar{F}(t)$ has decreasing hazard rate, we have $r(x) \geq$ $r(t) \forall x \leq t$; that is, $r(x) / r(t) \geq 1 \forall x \leq t$; then we have

$$
\int_{t}^{\infty}\left[\frac{r(x)}{r(t)}\right]^{\alpha} \frac{\bar{F}^{\alpha}(x)}{\bar{F}^{\alpha}(t)} d x \geq \int_{t}^{\infty} \frac{\bar{F}^{\alpha}(x)}{\bar{F}^{\alpha}(t)} d x
$$

which further implies the following: for all $0<\alpha<1$,

$$
\begin{aligned}
& \frac{1}{(1-\alpha)} \int_{t}^{\infty}\left[\frac{r(x)}{r(t)}\right]^{\alpha} \frac{\bar{F}^{\alpha}(x)}{\bar{F}^{\alpha}(t)} d x \\
& \geq \frac{1}{(1-\alpha)} \int_{t}^{\infty} \frac{\bar{F}^{\alpha}(x)}{\bar{F}^{\alpha}(t)} d x, \\
& \frac{1}{(\alpha-1)}+\frac{1}{(1-\alpha)} \int_{t}^{\infty}\left[\frac{r(x)}{r(t)}\right]^{\alpha} \frac{\bar{F}^{\alpha}(x)}{\bar{F}^{\alpha}(t)} d x \\
& \geq \frac{1}{(\alpha-1)}+\frac{1}{(1-\alpha)} \int_{t}^{\infty} \frac{\bar{F}^{\alpha}(x)}{\bar{F}^{\alpha}(t)} d x, \\
& \frac{1}{(\alpha-1)}\left(1-\int_{t}^{\infty}\left[\frac{r(x)}{r(t)}\right]^{\alpha} \frac{\bar{F}^{\alpha}(x)}{\bar{F}^{\alpha}(t)} d x\right) \\
& \geq \frac{1}{(\alpha-1)}\left(1-\int_{t}^{\infty} \frac{\bar{F}^{\alpha}(x)}{\bar{F}^{\alpha}(t)} d x\right) .
\end{aligned}
$$

Now using (50) and (11), we have

$$
\eta_{\alpha}(E ; t) \geq \eta_{\alpha}(X ; t), \quad \forall 0<\alpha<1 .
$$

Also when $\alpha>1$, using (51), we have

$$
\eta_{\alpha}(E ; t) \leq \eta_{\alpha}(X ; t), \quad \forall \alpha>1 .
$$

Similarly it follows that if $\bar{F}(t)$ has increasing hazard rate, then

$$
\eta_{\alpha}(E ; t) \leq(\geq) \eta_{\alpha}(X ; t), \quad \forall 0<\alpha<1 \quad(\alpha>1) .
$$

\section{Empirical Cumulative Tsallis Entropy}

Let $X_{1}, X_{2}, \ldots, X_{n}$ be nonnegative, absolutely continuous, independent, and identically distributed random variables with distribution function $F(x)$. According to (8), we define the empirical cumulative Tsallis entropy as

$$
\eta_{\alpha}\left(\widehat{F}_{n}\right)=\frac{1}{\alpha-1}\left(1-\int_{0}^{\infty}\left(\widehat{F}_{n}(x)\right)^{\alpha} d x\right)
$$

$$
\alpha>0, \alpha \neq 1,
$$

where

$$
\widehat{F}_{n}(x)=\frac{1}{n} \sum_{i=1}^{n} I_{\left\{X_{i} \leq x\right\}}, \quad x \in R,
$$

is the empirical distribution of the sample and $X_{(1)}<X_{(2)}<$ $\cdots<X_{(n)}$ are the order statistic. Equation (56) can be expressed as

$$
\eta_{\alpha}\left(\widehat{F}_{n}\right)=\frac{1}{\alpha-1}\left(1-\sum_{j=1}^{n-1} \int_{X_{(j)}}^{X_{(j+1)}}\left(\widehat{F}_{n}(x)\right)^{\alpha} d x\right) .
$$

Recalling that

$$
\widehat{F}_{n}(x)= \begin{cases}0, & x<X_{(1)}, \\ \frac{j}{n}, & X_{(j)} \leq x<X_{(j+1)}, j=1,2, \ldots, n-1 \\ 1, & x \geq X_{(n)}\end{cases}
$$

from (58), we get

$$
\eta_{\alpha}\left(\widehat{F}_{n}\right)=\frac{1}{\alpha-1}\left(1-\sum_{j=1}^{n-1} U_{(j+1)}\left(\frac{j}{n}\right)^{\alpha}\right),
$$

$$
\alpha>0, \alpha \neq 1,
$$

where

$$
U_{(i)}=X_{(i)}-X_{(i-1)}, \quad i=1,2, \ldots, n
$$

are the sample spacings (cf. $[9,28])$.

In the following example we study the empirical cumulative Tsallis entropy for exponentially distributed random samples.

Example 12. Let $X_{1}, X_{2}, \ldots, X_{n}$ be a random sample of exponentially distributed random variables with parameter 
TABle 1: Mean of empirical cumulative Tsallis entropy for different values of $n$ and $\alpha$.

\begin{tabular}{lccccccc}
\hline$n$ & $\alpha=0.25$ & $\alpha=0.5$ & $\alpha=0.75$ & $\alpha=0.99$ & $\alpha=1.01$ & $\alpha=1.25$ & $\alpha=1.5$ \\
\hline 10 & -8.63 & -13.43 & -27.64 & -706.51 & 707.69 & 28.83 & 14.65 \\
50 & -59.81 & -90.24 & -181.31 & -4549.44 & 4550.71 & 182.59 & 91.55 \\
100 & -125.56 & -188.86 & -378.55 & -9480.62 & 9481.90 & 379.84 \\
500 & -656.74 & -985.64 & -1972.13 & -49320.07 & 49321.36 & 1973.43 & 986.97 \\
1000 & -1322.48 & -1984.26 & -3969.36 & -99250.80 & 99252.09 & 3970.66 & 1985.59 \\
\hline
\end{tabular}

TABLE 2: Variance of empirical cumulative Tsallis entropy for different values of $n$ and $\alpha$.

\begin{tabular}{lccccccc}
\hline$n$ & $\alpha=0.25$ & $\alpha=0.5$ & $\alpha=0.75$ & $\alpha=0.99$ & $\alpha=1.01$ & $\alpha=1.25$ & $\alpha=1.5$ \\
\hline 10 & 2.46 & 5.03 & 18.45 & 10673.12 & 10606.53 & 15.77 & 3.66 \\
50 & 2.80 & 6.14 & 23.97 & 14664.52 & 14638.81 & 22.95 & 5.62 \\
100 & 2.86 & 6.33 & 24.98 & 15420.17 & 15404.57 & 24.35 \\
500 & 2.91 & 6.52 & 25.97 & 16179.85 & 16175.45 & 25.79 & 6.02 \\
1000 & 2.92 & 6.55 & 26.13 & 16300.87 & 16298.39 & 26.03 & 6.43 \\
\hline
\end{tabular}

$\lambda$. By Pyke [29], the sample spacings are independent, with $U_{(j+1)}$ exponentially distributed with parameter $\lambda(n-j)$. Hence from (60) we obtain the mean and variance of the empirical cumulative Tsallis entropy as follows:

$$
\begin{array}{r}
E\left(\eta_{\alpha}\left(\widehat{F}_{n}\right)\right)=\frac{1}{(\alpha-1)}\left[1-\sum_{j=1}^{n-1} \frac{1}{\lambda(n-j)}\left(\frac{j}{n}\right)^{\alpha}\right], \\
\alpha>0, \alpha \neq 1, \\
\operatorname{Var}\left(\eta_{\alpha}\left(\widehat{F}_{n}\right)\right)=\frac{1}{(\alpha-1)^{2}} \sum_{j=1}^{n-1} \frac{1}{\lambda^{2}(n-j)^{2}}\left(\frac{j}{n}\right)^{2 \alpha}, \\
\alpha>0, \alpha \neq 1 .
\end{array}
$$

Based on the empirical cumulative Tsallis entropy for random samples from exponential distribution with mean 1 , we tabulated the values for mean and variance in Tables 1 and 2, respectively. It may be observed from tabulated data that the mean of empirical cumulative Tsallis entropy, that is, $E\left(\eta_{\alpha}\left(\widehat{F}_{n}\right)\right)$, is decreasing for different values of $n$, whereas the variance of empirical cumulative Tsallis entropy, that is, $\operatorname{Var}\left(\eta_{\alpha}\left(\widehat{F}_{n}\right)\right)$, is increasing for different values of $n$.

\section{Conclusion}

The dynamic generalized information measure based on cumulative distribution function is more stable rather than the density function. In this paper, we proposed the dynamic cumulative residual Tsallis entropy which is found to be monotonic in nature. Based on the proposed DCRTE, we characterized some well known lifetime distributions such as exponential, Weibull, Pareto, and finite range distributions which play a vital role in reliability modeling. Here we proposed weighted dynamic cumulative residual Tsallis entropy and examine its application in relation to weighted and equilibrium models. Finally, we introduce empirical cumulative Tsallis entropy for empirical samples.

\section{Conflict of Interests}

The authors declare that there is no conflict of interests regarding the publication of this paper.

\section{Acknowledgments}

The authors are thankful to the referees for their valuable suggestions which significantly improved the presentation. Nitin Gupta would like to thank Department of Science and Technology, India, for a research grant (SR/FTP/MS022/2011) and NBHM, DAE, India, for a research grant (NBHM/R.P.21/2012/Fresh/1744).

\section{References}

[1] C. E. Shannon, "A mathematical theory of communication," Bell System Technical Journal, vol. 27, pp. 379-423, 1948.

[2] N. Ebrahimi, "How to measure uncertainty in the residual life time distribution," Sankhyā Series A, vol. 58, no. 1, pp. 48-56, 1996.

[3] M. Rao, Y. Chen, B. C. Vemuri, and F. Wang, "Cumulative residual entropy: a new measure of information," IEEE Transactions on Information Theory, vol. 50, no. 6, pp. 1220-1228, 2004.

[4] M. Asadi and N. Ebrahimi, "Residual entropy and its characterizations in terms of hazard function and mean residual life function," Statistics \& Probability Letters, vol. 49, no. 3, pp. $263-$ 269, 2000.

[5] M. Asadi, N. Ebrahimi, and E. S. Soofi, "Dynamic generalized information measures," Statistics and Probability Letters, vol. 71, no. 1, pp. 85-98, 2005.

[6] M. Rao, "More on a new concept of entropy and information," Journal of Theoretical Probability, vol. 18, no. 4, pp. 967-981, 2005.

[7] N. Drissi, T. Chonavel, and J. M. Boucher, "Generalized cumulative residual entropy for distributions with unrestricted supports," Research Letters in Signal Processing, vol. 2008, Article ID 790607, 5 pages, 2008.

[8] R. C. Gupta, "Some characterization results based on residual entropy function," Journal of Statistical Theory and Applications, vol. 8, no. 1, pp. 45-59, 2009. 
[9] A. Di Crescenzo and M. Longobardi, "On cumulative entropies," Journal of Statistical Planning and Inference, vol. 139, no. 12, pp. 4072-4087, 2009.

[10] J. Navarro, Y. del Aguila, and M. Asadi, "Some new results on the cumulative residual entropy," Journal of Statistical Planning and Inference, vol. 140, no. 1, pp. 310-322, 2010.

[11] R. C. Gupta and H. C. Taneja, "Entropy and residual entropy functions and some characterization results," Pakistan Journal of Statistics and Operation Research, vol. 8, no. 3, pp. 605-617, 2012.

[12] H. C. Taneja and V. Kumar, "On dynamic cumulative residual inaccuracy measure," in Proceedings of the World Congress on Engineering, vol. 1, pp. 153-156, 2012.

[13] M. Asadi and Y. Zohrevand, "On the dynamic cumulative residual entropy," Journal of Statistical Planning and Inference, vol. 137, no. 6, pp. 1931-1941, 2007.

[14] M. Abbasnejad, N. R. Arghami, S. Morgenthaler, and G. R. M. Borzadaran, "On the dynamic survival entropy," Statistics and Probability Letters, vol. 80, no. 23-24, pp. 1962-1971, 2010.

[15] S. M. Sunoj and M. N. Linu, "Dynamic cumulative residual Renyi's entropy," Statistics, vol. 46, no. 1, pp. 41-56, 2012.

[16] V. Kumar and H. C. Taneja, "Some characterization results on generalized cumulative residual entropy measure," Statistics \& Probability Letters, vol. 81, no. 8, pp. 1072-1077, 2011.

[17] A. Renyi, "On measures of entropy and information," in Proceedings of the 4th Berkeley Symposium on Mathematics, Statistics and Probability, J. Neyman, Ed., vol. 1, pp. 547-561, University of California Press, Berkeley, Calif, USA, 1961.

[18] C. Tsallis, "Possible generalization of Boltzmann-Gibbs statistics," Journal of Statistical Physics, vol. 52, no. 1-2, pp. 479-487, 1988.

[19] C. Beck, "Generalised information and entropy measures in physics," Contemporary Physics, vol. 50, no. 4, pp. 495-510, 2009.

[20] N. Gupta and R. K. Bajaj, "On partial monotonic behaviour of some entropy measures," Statistics and Probability Letters, vol. 83, no. 5, pp. 1330-1338, 2013.

[21] J. Cartwright, "Roll over, boltzmann," Physics World, vol. 27, no. 5, pp. 31-35, 2014.

[22] W. J. Hall and J. A. Wellner, "Mean residual life," in Statistics and Related Topics, M. Csorgo, D. A. Dawsan, J. N. K. Rao, and A. K. Md. E. Saleh, Eds., pp. 169-184, North-Holland, Amsterdam, The Netherlands, 1981.

[23] G. P. Patil and C. R. Rao, "The weighted distributions: a survey of their applications," in Applications of Statistics, pp. 383-405, North-Holland, 1977.

[24] R. C. Gupta and S. N. U. A. Kirmani, "The role of weighted distributions in stochastic modeling," Communications in Statistics. Theory and Methods, vol. 19, no. 9, pp. 3147-3162, 1990.

[25] N. U. Nair and S. M. Sunoj, "Form-invariant bivariate weighted models," Statistics, vol. 37, no. 3, pp. 259-269, 2003.

[26] A. Di Crescenzo and M. Longobardi, "On weighted residual and past entropies," Scientiae Mathematicae Japonicae, vol. 64, no. 2, Article ID 0703489, pp. 255-266, 2006.

[27] S. S. Maya and S. M. Sunoj, "Some dynamic generalized information measures in the context of weighted models," Statistica, vol. 68, no. 1, pp. 71-84, 2008.

[28] A. Di Crescenzo and M. Longobardi, "Neuronal data analysis based on the empirical cumulative entropy," in Computer Aided Systems Theory-EUROCAST 2011, vol. 6927 of Lecture Notes in Computer Science, pp. 72-79, Springer, Berlin, Germany, 2012.
[29] R. Pyke, "Spacings," Journal of the Royal Statistical Society B. Methodological, vol. 27, pp. 395-449, 1965. 


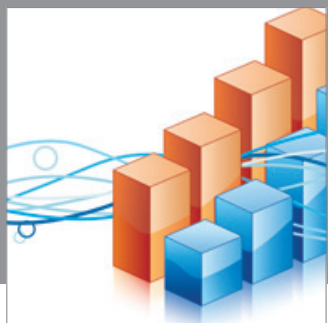

Advances in

Operations Research

mansans

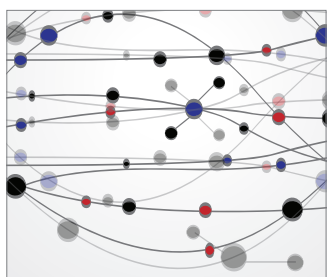

The Scientific World Journal
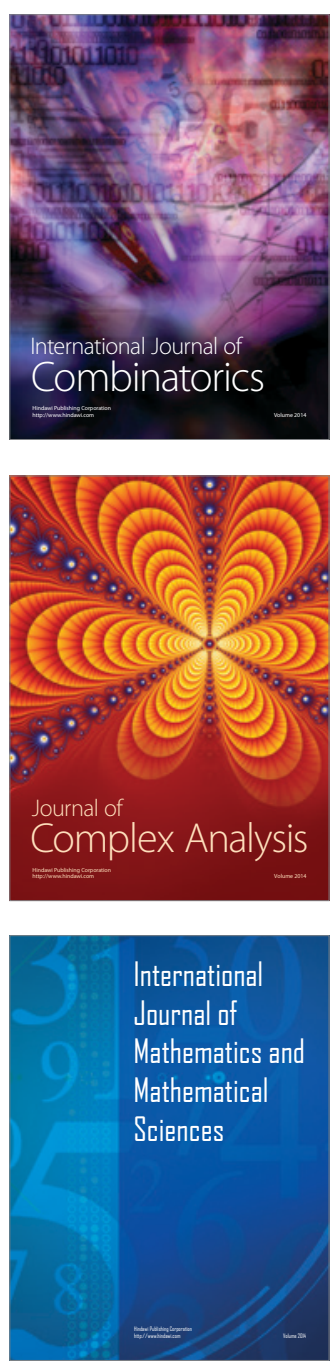
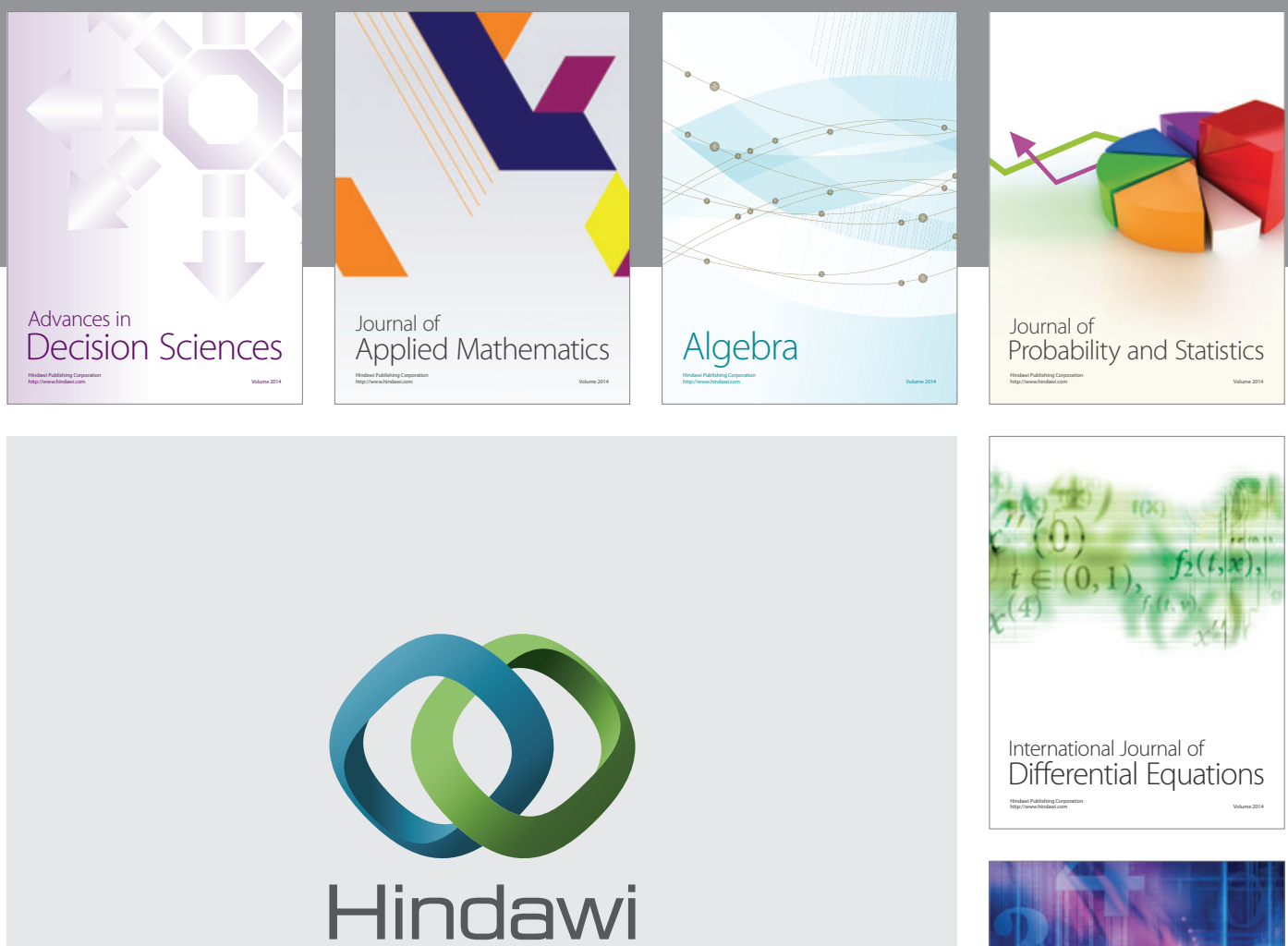

Submit your manuscripts at http://www.hindawi.com
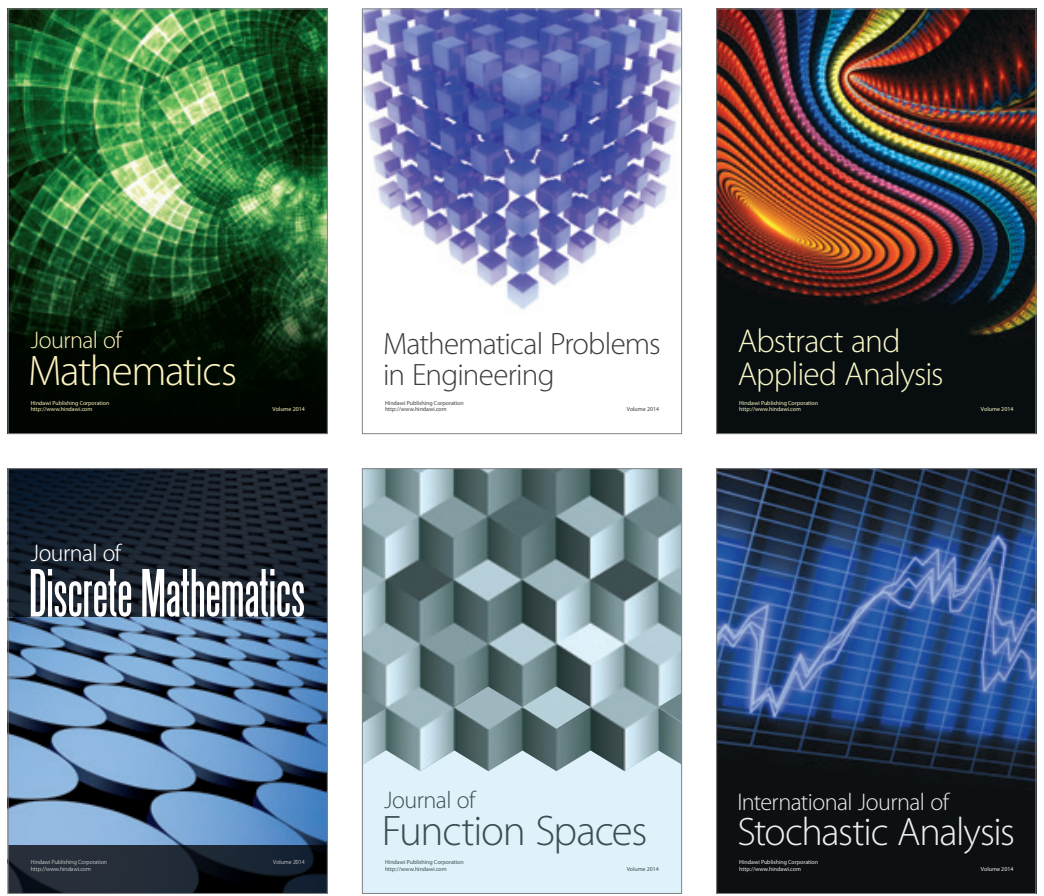

Journal of

Function Spaces

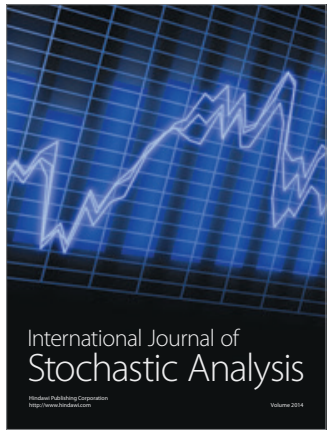

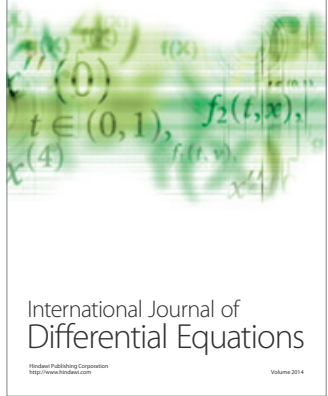
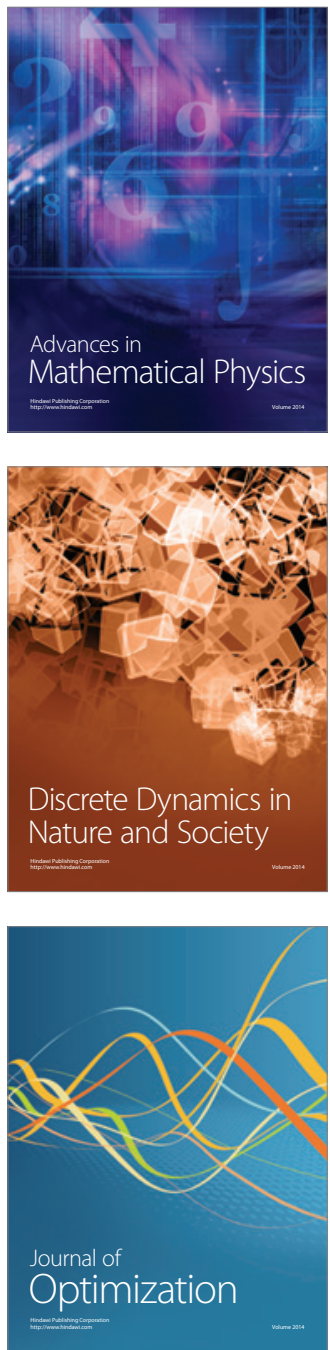